\title{
Introducción
}

\section{Estado del arte en imaginarios y representaciones: un mapa necesario para buscar caminos investigativos en Iberoamérica}

Felipe Aliaga

María LiLy Maric

Cristhian URIBE

7 xiste una serie de fenómenos que se enmarca en procesos de consEtrucción de sentido compartido en donde operan figuras simbólicas, estructuras de pensamiento, memorias colectivas, formas arquetípicas, entre otros elementos que van construyendo, de-construyendo y haciendo circular lo que se ha denominado como imaginarios y representaciones sociales. Hay múltiples investigaciones en el mundo que se adscriben a estos conceptos como elementos que pueden ser analizados desde diferentes enfoques teóricos y metodológicos; esto ha derivado en la existencia de una serie de pensadores que ofrecen pautas para investigadores y grupos de investigación en el mundo, que se dedican a estas materias y que han publicado innumerables obras, fruto del trabajo desde diferentes disciplinas.

Reconocidos autores como Emile Durkheim, Cornelius Castoriadis, Gilbert Durand, Michel Maffesoli, Manuel Antonio Baeza, Enrique Carretero, Armando Silva, Serge Moscovici, Denise Jodelet, Stuart Hall, entre otros, han abordado los imaginarios y las representaciones. Esto ha dado como resultado una gran cantidad de material teórico 
que sirve de base para la investigación en diferentes campos científicos. Se han compartido enfoques y creado novedosas combinaciones interdisciplinarias para la interpretación de los fenómenos sociales. Este proceso ha dado origen a múltiples grupos de investigación, a modo de ejemplo: Centro de estudios sobre el imaginario (Argentina), ${ }^{1}$ Centre de recherche sur l'imaginaire (Bélgica), ${ }^{2}$ Associação Ylê Setí do Imaginário (Brasil), ${ }^{3}$ Groupe de recherche sur les imaginaires politiques en Amérique latine (Gripal) (Canadá), ${ }^{4}$ Grupo Concepción de Estudios Sobre Imaginarios Sociales (GCEIS) (Chile), Grupo Prácticas culturales, imaginarios y representaciones (Colombia), ${ }^{5}$ Poder y representación. Transferencias culturales en la época moderna (España), ${ }^{6}$ Centre de recherche sur l'imaginaire (Francia), ${ }^{7}$ Centrul de Cercetare a Imaginarului (PHAntasma) (Rumanía), ${ }^{8}$ Cattedra Unesco Cultural and comparative study on imaginary (Italia). ${ }^{9}$

Este amplio panorama científico evidencia que existe un gran interés por estas materias, ante lo cual surge la necesidad de conocer las investigaciones que se están llevando a cabo, su pluralidad de objetos de estudio, perspectivas teóricas y metodológicas, y formas de triangulación, entendiendo esta como la describe María Cea D’Ancona, “a) Triangulación de datos. b) Triangulación de investigadores. c) Triangulación teórica, d) Triangulación metodológica

1 Para más información ver en: http://www.ciencias.org.ar/listacategoriasproductos .asp ?idCategory $=920 \&$ tipo $=0 \&$ urldes $=\&$ descurl $=$

2 Para más información ver en: http://www.uclouvain.be/centre-recherche-imaginaire.html

3 Para más información ver en: http://www.yle-seti-imaginario.org/

4 Para más información ver en: http://www.gripal.ca/

5 Para más información ver en: http://www.unal.edu.co/ces/index.php/ investigacion/estructura/grupos/217

6 Para más información ver en: http://www.ub.edu/poderirepresentacions/?lang=es

7 Para más información ver en: http://cri.u-grenoble3.fr/

8 Para más información ver en: http://phantasma.lett.ubbcluj.ro/

9 Para más información ver en: http://www.iulm.it/wps/wcm/connect/masterit/ cattedra+unesco/home 
—intramétodo y entre métodos-" (Cea, 2001, p. 49). Se trata de realizar un estado del arte sobre la investigación en torno a los imaginarios y las representaciones, el cual se asume desde la perspectiva de Absalón Jiménez "estamos hablando de la necesidad hermenéutica de remitirnos a textos que a su vez son expresiones de desarrollos investigativos, dados desde diversas percepciones de las ciencias sociales y escuelas de pensamiento" (2004, p. 32).

De esta manera es importante conocer este quehacer investigativo para ver por dónde se está moviendo la ciencia social en estos campos de estudio, y cuáles son las tendencias investigativas que se están estableciendo en los últimos años, en cuanto "En los estados del arte se establece la necesidad de revisar y cimentar los avances investigativos realizados por otros, aclarar rumbos, contrastar enunciados provisionales y explorar nuevas perspectivas de carácter inédito, ya sea con respecto a los objetos de estudio, sus formas de abordaje, percepciones, paradigmas y metodologías, incluyendo el tipo de respuestas al que se ha llegado" (Jiménez, 2004, p. 33).

Este estudio se contextualiza en el espacio geográfico iberoamericano, con la intención de indagar en la existencia de las principales temáticas y campos de estudio, permitiendo conocer distancias o similitudes en la investigación realizada en los distintos países. Igualmente, este proyecto se articula a la Red Iberoamericana de Investigación en Imaginarios y Representacione (RIIR), ${ }^{10}$ desde donde se hace importante llevar a cabo estudios de esta naturaleza, y responder a la pregunta ¿Cómo se está desarrollando la investigación en torno a los imaginarios y las representaciones en Iberoamérica?, cuestión que hasta el momento no ha sido abordada.

De esta manera, el objetivo general de este proyecto fue identificar la investigación en torno a los imaginarios y las representaciones sociales en Iberoamérica. Los objetivos específicos fueron establecer tipos de investigación en imaginarios y representaciones sociales en los diferentes países iberoamericanos, determinar triangulaciones entre enfoques teóricos e investigación aplicada en imaginarios y representaciones

10 Ver más en: https://imaginariosyrepresentaciones.wordpress.com/ 
sociales y enunciar las áreas y tendencias de la investigación en imaginarios y representaciones sociales en Iberoamérica.

Es estudiando las mismas investigaciones que se pueden rastrear campos teóricos y metodológicos, como también se puede rastrear la interdisciplina, ya que los trabajos pueden estar ofreciendo pistas de traspaso de fronteras disciplinarias. Las investigaciones abordan un tema utilizando varios encuadres o perspectivas (Hernández Sampieri y otros, 2010); como indican Aliaga y Pintos (2012, p. 11), "se asume que la ciencia social y las demás ciencias, son de por sí limitadas a sus propias definiciones, lo cual deja de manifiesto la necesidad fundamental del diálogo entre las disciplinas, para poder alcanzar un tratamiento lo más amplio posible de los fenómenos a estudiar”. Encontrar los elementos de interdisciplinariedad o proponer los caminos puede ser uno de los importantes frutos de esta investigación, pues "la investigación hoy en día necesita de trabajo multidisciplinario, lo cual contribuye a que se realice en equipos integrados por personas con intereses y aproximaciones metodológicas diversas, que refuerza la necesidad de usar diseños multimodales" (Creswell, 2009, citado en Hernández y otros, 2010, p. 549).

Este libro abre pautas a la comprensión de la interrelación en las formas de la investigación científica en las ciencias sociales, en tanto que "cabe apuntar que las maneras de investigar cambian a medida que se transforma la composición de la sociedad, ante lo cual no hay teorías o modelos que podamos definir como verdades o dogmas científicos, ya que pueden tener operatividad en determinados momentos o servir como esquemas de análisis para algunas cuestiones" (Aliaga y Pintos, 2012, p. 11). En este sentido, esta investigación ayudará a encontrar causas o efectos que se están tratando de identificar, analizar o describir en diferentes estudios; pudiendo observar distintas posibilidades de acercamiento a fenómenos sociales, considerando que comprender la realidad es un proceso complejo, dada la condición multi-causal y multi-efecto de todo fenómeno, siguiendo la idea de causalidad de Niklas Luhmann en donde "la causalidad es un esquema de observación del mundo: siempre es posible buscar más causas de las causas; y de los efectos buscar más efectos, por ejemplo, efectos colaterales. El esquema de causalidad tiene límites 
fundamentales, ya que nunca se podría incluir, en él, la totalidad de las causas en el mundo" (1996, p. 79).

Este estado del arte ayudará a comprender por dónde se están moviendo los intereses investigativos, dentro de la pluralidad de causas y efectos que se pueden ver descritos en las diferentes investigaciones; como apunta Absalón Jiménez "los estados del arte no se acercan a la principal fuente del conocimiento social, que es la realidad, la experiencia como tal y la cotidianidad; más bien, parten de un producto de lo dado y acumulado por las ciencias sociales" (Jiménez, 2004, p. 31). El autor indica que los estados del arte contribuyen a identificar nuevos caminos para la investigación y no partir de la nada.

$\mathrm{Al}$ enfocarse este estudio en Iberoamérica, se enmarca en lo que la Organización de Estados Iberoamericanos (OEI) ha denominado “Espacio Iberoamericano de Conocimiento” el cual según José García y Marisol González (2011, p. 32) "tiene por objetivo el desarrollo de un espacio interactivo y de colaboración en los ámbitos de educación superior y la investigación como rectores del conocimiento científico y tecnológico, que debe estar articulado con el desarrollo y la innovación". De esta manera esta investigación puede ofrecer material para aumentar las posibilidades de investigar en Iberoamérica en torno a los imaginarios y las representaciones, como driría Jiménez "el estado del arte representa el primer insumo, y tal vez el más importante, para la iniciación de cualquier tipo de investigación” (2004, p. 35).

El origen de este proyecto surge con motivo de la visita del coordinador general de la Red Iberoamericana de Investigación en Imaginarios y Representaciones (RIIR), ${ }^{11}$ Felipe Aliaga, al Instituto de Estudios Bolivianos (IEB) de la Universidad Mayor de San Andrés (UMSA) ${ }^{12}$, en La Paz-Bolivia, en la cual se formalizó el ingreso del Ієв como la institución representante de Bolivia en la red. En esa ocasión se acordó que la representante del IEB sería María Lily Maric. En este trabajo de colaboración internacional se comienza a desarrollar el proyecto

11 Para mayor información consultar en: https://imaginariosyrepresentaciones. wordpress.com/

12 La visita se realizó del 23 al 25 de septiembre de 2015. 
para escribir el primer libro sobre la investigación en Iberoamérica en torno a los imaginarios y representaciones sociales, con la finalidad de ir identificando las principales líneas de investigación y las tendencias en los diferentes países vinculados a la red, así comienza un intenso proceso de coordinación para hacer real esta iniciativa.

Es importante destacar que, en la actualidad, no existe un estado del arte de esta envergadura, que presente una amplia descripción de la investigación que se ha llevado o está siendo llevada a cabo. Este proyecto reúne para su ejecución investigadores de nueve países, de diferentes disciplinas y universidades, constituyéndose como un proyecto internacional, interdisciplinario e interinstitucional.

No se pueden desconocer algunos esfuerzos anteriores de sistematización de la investigación en relación a esta materia, un esbozo de la investigación sobre los imaginarios sociales lo constituye el artículo de Felipe Aliaga y Juan Luis Pintos (2012), denominado "La investigación en torno a los imaginarios sociales. Un horizonte abierto a las posibilidades", el cual es la introducción al extraordinario monográfico Investigación social en torno a los imaginarios sociales, publicado en la Revista de Investigaciones políticas y sociológicas (RIPS), coordinado por los mismos autores. En ese trabajo se describe brevemente una serie de grupos de investigación dedicados al estudio de lo imaginario, dentro de ellos los que pertenecen a la federación internacional Groupement Coordonné des Centres de Recherche sur l'Imaginaire (Grevo-CRI). También identifican dos corrientes intelectuales, la francesa y la corriente iberoamericana. El trabajo concluye "el sentido que tiene esta introducción es abrir una vía que hasta ahora no ha sido transitada y animar a los diferentes investigadores implicados a seguir este u otro esbozo ampliando y profundizando las referencias aquí iniciadas" (Aliaga y Pintos, 2012, p. 17).

En 2012, la investigadora Lidia Girola publica el capítulo denominado "Representaciones e Imaginarios sociales. Tendencias recientes en la investigación”, en el libro editado por Enrique De la Garza y Gustavo Leyva, Tratado de metodología de las ciencias sociales: perspectivas actuales. Girola realiza algunas distinciones entre las nociones de representaciones e imaginarios sociales; abordando el concepto de representación colectiva desde Emile Durkheim hasta el de representación 
social de Serge Moscovici. Girola menciona que existen diversas propuestas metodológicas para el análisis de las representaciones sociales, y que del pensamiento de Moscovici han surgido diversas corrientes, por ejemplo la "Escuela clásica", la "Escuela de Aix-en-Provence", la "Escuela de Ginebra". Así también, hay investigadores en España y América Latina, de los cuales se destacan las aportaciones del Grupo de Trabajo de Imaginarios Latinoamericanos.

Lidia Girola destaca particularmente en México la existencia de diversos grupos: "La complejidad y riqueza de los estudios recientes sobre representaciones sociales hacen sumamente difícil hacer aquí un recuento exhaustivo del estado del arte en el tema, por lo que me limito a decir que existen estudios sobre representaciones sociales del cuerpo, el trabajo, el policía auxiliar, el desempleo (Rodríguez-Salazar, García-Curiel, 2007); la escuela, mitos de origen en comunidades rurales (Arruda, de Alba, 2007); el papel de la enfermera en la sociedad actual (Araya, 2002) y un largo etcétera. Las citas y sitios en Internet aumentan exponencialmente, día con día” (Girola, 2012, p. 410).

Girola hace una genealogía del concepto de imaginarios sociales desde los años setenta con autores como Emile Durkheim, Cornelius Castoriadis, Benedict Anderson, John R. Searle; hasta los años noventa en donde a nivel Iberoamericano la problemática de lo imaginario es analizada por Juan Luis Pintos desde el Grupo Compostela de Estudios sobre Imaginarios Sociales; así como por la perspectiva fenomenológica de Manuel Antonio Baeza; Charles Taylor con los imaginarios sociales modernos; Daniel Hiernaux y la misma autora, sobre los imaginarios urbanos.

Margarita Zires y Yael Merino en 2007 publican en la revista Versión, en el monográfico Comunicación: imaginarios y representaciones sociales, el artículo "Referencias bibliográficas sobre el estudio del imaginario social”. En ese trabajo los autores presentan una serie de libros en español, en relación a las corrientes de pensamiento que se generaron a partir de Gilbert Durand y Cornelius Castoriadis, afirmando que "dichas corrientes se caracterizan por conceder una importancia fundamental a la facultad de la imaginación del ser humano y destacan el papel constitutivo de lo imaginario en la construcción de la realidad social"(Zires y Merino, 2007, p. 341). Se incluyen referencias 
desde diferentes disciplinas que permiten comprender la configuración de la sociedad a partir de lo imaginario.

En la misma revista mencionada anteriormente Silvia Gutiérrez (2007) publica: "Las representaciones sociales: Panorama bibliográfico". La autora indica que la noción de representación ocupa un lugar importante en las ciencias sociales y la teoría de las representaciones ha permitido realizar muchos estudios, "las líneas de investigación de esta teoría son numerosas y variadas y si bien en la psicología social es donde más ejemplos encontramos, actualmente está teniendo una repercusión importante en varios campos de investigación" (Gutiérrez, 2007, p. 315). Ofrece una revisión bibliográfica en lengua española organizada por temas, en los que se incluyen: listado de artículos por temas; aspectos teóricos; propuestas metodológicas; cultura/identidad; género; educación/contexto escolar; política y movimientos sociales; comunicación; lenguaje/discurso; salud pública, salud mental; trabajo y prácticas profesionales; problemas sociales; pobreza; SIDA; medio ambiente; violencia; además de presentar los formatos como entrevistas; libros; tesis de grado; maestría; doctorado y sitios de Internet.

Maricela Perera en 2002 publica A propósito de las representaciones sociales. Apuntes teóricos, trayectoria y actualidad, en el que da a conocer una serie de abordajes teóricos del concepto desde Durkheim, Moscovici y Jodelet, esta última será quien sistematice las ideas de Moscovici e impulse una corriente desde París con un abordaje desde la etnometodología; también describe la existencia de estudios realizados en Suiza por Willem Doise en cuanto la inserción de las estructuras sociales en las representaciones, quienes trabajan desde una perspectiva estadística; y la corriente francesa liderada por Jean-Claude Abric desde una dimensión cognitivo-estructural en cuanto Teoría del Núcleo Central, aplicado en entrevistas.

La autora hace un repaso por una serie de funciones, fuentes, estructuras, contenidos y mecanismos de formación de las representaciones sociales. Incluye un acápite sobre los estudios en la actualidad, en donde indica que "El volumen y ascendente cantidad de estudios que en el mundo se realizan y que en forma de libros, artículos en revistas, tesis de grado, maestría y doctorado, presentaciones en eventos, etc., recogen el quehacer en este campo. Hacer un levantamiento en 
tal sentido constituye en sí mismo un estudio serio y minucioso; muy lejos de nuestras modestas posibilidades de acceder a la literatura internacional y aquí justamente situamos el inconveniente que personalmente más nos impacta” (Perera, 2002, p. 26). Sin embargo, menciona la Conferencia Internacional sobre Representaciones Sociales que se ha realizado en diferentes países, incluyendo Brasil (1994) y México (1998), en donde se puede encontrar temas como: la ciencia, el saber académico/pensamiento o conocimiento popular; salud/enfermedad; el desarrollo humano; el campo educativo; el trabajo; participación/ exclusión social; y la cuestión comunitaria. Indica que específicamente en Cuba en la década de los años noventa se ha investigado sobre salud, género-familia, vida cotidiana y el área político social.

Cabe mencionar que en 1997 Denis Jodelet y Jocelyne Ohana, publican en el libro Les représentations sociales, un capítulo denominado "Bibliographie générale sur les représentations sociales", en donde publican una bibliografía hasta 1996, fundamentalmente de libros y artículos en torno a las representaciones sociales, que provienen en su mayoría de la psicología social.

La revisión de los trabajos mencionados permite afirmar que nos encontramos frente a un panorama amplio, pero que es posible abordar en un esfuerzo conjunto por identificar la investigación que se haya o se esté desarrollando en los diferentes países de Iberoamérica.

En el trabajo de elaboración del estado del arte se realizó una recuperación, en los diferentes países del contexto iberoamericano, de las experiencias de investigación aplicada que se hayan llevado a cabo, tomando como referencia las sugerencias de Absalón Jiménez, en cuanto:

[...] primero desarrollar una contextualización de la temática clasificando el tipo de textos, autores, metodologías, marcos de referencia conceptual y niveles conclusivos de las diversas investigaciones; en segundo lugar dicha ubicación de información nos permite establecer una clasificación del tipo de trabajos consultado sus convergencias y divergencias conceptuales, metodológicas y conclusivas; en tercer lugar, se sugiere una categorización de los 
trabajos partiendo de las categorías internas de cada uno de los textos, lo que nos permite establecer el aporte que ofrecen desde la panorámica sociocultural en el área de investigación que se desarrolla. (Calvo, 2003, citado en Jiménez, 2004, p. 37).

El enfoque metodológico puede variar en cuanto a la forma de construcción del estado del arte. Al respecto, Jiménez (2004) indica que no hay una metodología única para la construcción de un estado del arte: sin embargo, se debe tener claridad en la pregunta, sistematicidad en la lectura de los textos, en la captura y utilización de la información contenida, así como la confiabilidad de quien los interpreta. El acercamiento a los textos se debe realizar desde la hermenéutica, ya que los textos "constituyen formas de abordaje previas que contienen análisis, conceptos, categorías, hipótesis y hasta teorías" (Jiménez, 2004, p. 39). Este proceso incluye un balance de textos en donde hay que considerar condiciones culturales, políticas y sociales.

En este libro se articulan múltiples formas metodológicas; consta de nueve capítulos que componen este "mapa necesario para buscar caminos investigativos en Iberoamérica”, provenientes de investigadores de Argentina, Bolivia, Brasil, Colombia, Chile, Ecuador, España, México y Venezuela. El abordaje responde a su historicidad científica y al interés o necesidad de aplicar diferentes perspectivas teóricas y metodológicas en relación al imaginario y las representaciones sociales. Esperamos que esta obra se constituya en un valioso aporte a la investigación en la materia.

\section{Referencias bibliográficas}

Aliaga, F. y Pintos, J. (2012). La investigación en torno a los imaginarios sociales. Un horizonte abierto a las posibilidades. Revista de Investigaciones políticas y sociológicas (RIPS), 11 (2).

Araya, S. (2002). Las representaciones sociales: Ejes teóricos para su discusión. Costa Rica: Flacso.

Baeza, M. (2000). Los caminos invisibles de la realidad social. Ensayo de sociología profunda sobre imaginarios sociales. Santiago de Chile, Chile: RIL. 
Carretero, E. (2003). Postmodernidad e imaginario. Una aproximación teórica. Foro Interno, 3. En línea. Recuperado de : http://dialnet.unirioja. es/servlet/articulo?codigo $=1334283>$

Carretero, E. (2011). El orden social en la posmodernidad. Ideología e imaginario social. Barcelona, España: Erasmus.

García J. y González, M. (2011). El espacio iberoamericano del conocimiento. Una visión desde la universidad latinoamericana y caribeña. Revista Iberoamericana de Educación, 57, 31-51

Girola, L. (2012). Representaciones e Imaginarios sociales. Tendencias recientes en la investigación. En De la Garza, E. y Leyva, G. (Eds.). Tratado de metodología de las ciencias sociales: perspectivas actuales. Ciudad de México, México: Fondo de Cultura Económica.

Gutiérrez, S. (2007). Las representaciones sociales: Panorama bibliográfico. Versión, 19.

Hernández, R.; Fernández, C. y Baptista, M. (2010). Metodología de la investigación. Quinta edición. Ciudad de México, México: McGraw Hill Interamericana.

Jiménez, A. (2004). El estado del arte en la investigación en las ciencias sociales. En Jiménez, A. y Torres, A. (Comp.). La práctica investigativa en ciencias sociales. Bogotá, Colombia: UPN.

Jodelet, D. (2007). Imbricaciones entre representaciones sociales e intervención. En Rodríguez, T. y García, M. (Coord.). Representaciones sociales. Teoría e investigación. Guadalajara, México: Editorial cucsH-udG.

Jodelet, D. et Ohana, J. (1997). Bibliographie générale sur les représentations sociales. Dans Jodelet, D. (Coord.). Les représentations sociales. París, Francia: PUF.

Luhmann, N. (1996). Introducción a la teoría de sistemas. (Lecciones publicadas por Javier Torres Nafarrate). México: Universidad Iberoamericana/ Anthropos/ ITESO.

Moscovici, S. (1979). El psicoanálisis, su imagen y su público. Buenos Aires, Argentina: Huemul.

Perera, M. (2003). A propósito de las representaciones sociales: apuntes teóricos, trayectoria y actualidad. La Habana, Cuba: cips.

Pintos, J. (1995). Los imaginarios Sociales. La nueva construcción de la realidad social. Cantabria, Madrid, España: Sal Terrae/ "Fe y Secularidad". 
Pintos, J. (2004). Inclusión - Exclusión. Los imaginarios sociales de un proceso de construcción social. SEMATA. Ciencias Sociales y Humanidades, 6, 17-52.

Silva, A. (2006). Imaginarios urbanos. (Quinta edición). Bogotá, Colombia: Arando Editores.

Zires, M.; Merino, Y. (2007). Referencias bibliográficas sobre el estudio del imaginario social. Versión, 19. 